\title{
THE DRAG AND SPHERICITY INDEX OF A SPINDLE*
}

\author{
By D. M. STASIW, F. B. COOK, M. C. DETRAGLIA And L. C. CERNY \\ (Masonic Medical Research Laboratory and Utica College of Syracuse University, Utica)
}

Introduction. Several years ago, Payne and Pell [1, 2, 3] published a series of articles pertaining to the Stokes flow of a viscous, incompressible fluid about a body in which the flow is two-dimensional or has radial symmetry. Some of the shapes of bodies that were considered were a lens, a torus, a sphere, and oblate and prolate spheroids. Using this procedure, the differential equation to be satisfied in the flow region is found to be

$$
L_{-1}^{2} \psi_{1}=0
$$

where

$$
L_{-1}=\frac{\partial^{2}}{\partial x^{2}}+\frac{\partial^{2}}{\partial r^{2}}-\frac{1}{r} \frac{\partial}{\partial r} .
$$

If the trace of the boundary of the body is called $C$, then the condition of vanishing velocity on $C$ can be stated in the form

$$
\psi_{1}=0, \quad \partial \psi_{1} / \partial n=0, \text { on } C .
$$

Here $n$ is the unit normal to $C$ exterior to the body.

Recently, our interest has been in the general area of the flow of diseased states of blood. The above papers have been extremely useful in a problem of current medical concern, sickle-cell anemia. The red cells in this diseased state approximate lens, sickles, hemispheres and spindles. It is the purpose of this communication to show the calculation of the flow about a spindle.

Flow about a spindle. A convenient set of coordinates to represent a spindle are the bipolar coordinates $(\varphi, \eta)[4]$. In terms of these coordinates

$$
\begin{gathered}
x=\frac{b \sinh \eta}{\cosh \eta-\cos \varphi}, \\
r=\frac{b \sin \varphi}{\cosh \eta-\cos \varphi}, \\
x^{2}+r^{2}-2 b r \cot \varphi=b^{2},
\end{gathered}
$$

with $\varphi>\pi / 2 ; r \geq 0$. The calculation of the drag, $P$, can be made by using

$$
P=8 \pi \mu \lim _{\rho \rightarrow \infty}\left(\rho \psi_{1} / r^{2}\right) .
$$

* Received August 24, 1973. This work was partially supported by a grant from the Public Health Service No. HL 13228. 
In this equation $\rho=\left(x^{2}+r^{2}\right)^{1 / 2}, \mu$ is the coefficient of viscosity of the suspending fluid and $\psi_{1}$ is a solution to

$$
L_{-1}^{2} \psi_{1}=0 .
$$

Payne and Pell [3] suggest that the drag can best be evaluated from

$$
P=8 \pi b \mu U \int_{0}^{\infty} \frac{F(\alpha)}{\cosh \alpha \pi} d \alpha,
$$

where

$$
F(\alpha)=\int_{t_{0}}^{1} K_{\alpha}(-\tau) K_{\alpha}{ }^{(2)}(\tau) d \tau / \int_{t_{0}}^{1} K_{\alpha}(\tau) K_{\alpha}{ }^{(2)}(\tau) d \tau .
$$

In Eq. (9) $K_{\alpha}(\tau)$ is known as the conal function (5) and defined as

$$
K_{\alpha}(\tau)=P_{i \alpha-1 / 2}(\tau)
$$

and

$$
K_{\alpha}{ }^{(n)}(\tau) \equiv d^{n} K_{\alpha}(\tau) / d \tau^{n},
$$

where $P(\tau)$ is the Legendre function, and $\tau=\cos \varphi$.

To date, the drag for a spindle has not been determined, although Payne and Pell suggest that certain tables should facilitate the computation [6, 7]. However, in examining these tables, it was found that they were not adequate with regard to the choice of the angle $\varphi$ or with ease of determining the derivatives of the conal functions. To overcome these difficulties the approach that follows was finally used.

The functions $B(\alpha)$ and $A(\alpha)$ are evaluated from the boundary conditions of the problem and can be written as

$$
\begin{aligned}
& A(\alpha)=\frac{2^{1 / 2}}{\Omega \cosh \alpha \pi}\left[K_{\alpha}\left(-t_{0}\right) K_{\alpha}^{(1)}\left(t_{0}\right)-K_{\alpha}{ }^{(1)}\left(-t_{0}\right) K_{\alpha}\left(t_{0}\right)\right], \\
& B(\alpha)=\frac{2^{1 / 2}}{\Omega \cosh \alpha \pi}\left[t_{0} K_{\alpha}^{(1)}\left(t_{0}\right) K_{\alpha}^{(1)}\left(-t_{0}\right)-K_{\alpha}\left(t_{0}\right) \frac{d}{d t_{0}}\left(t_{0} K_{\alpha}^{(1)}\left(t_{0}\right)\right]\right.
\end{aligned}
$$

where $t_{0}=\tau_{0}=\cos \varphi_{0}$ and

$$
\Omega=t_{0}\left[K_{\alpha}{ }^{(1)}\left(t_{0}\right)\right]^{2}-K_{\alpha}\left(t_{0}\right) \frac{d}{d t_{0}}\left(t_{0} K_{\alpha}{ }^{(1)}\left(t_{0}\right)\right)
$$

The value of $t_{0}$ defines the shape of the spindle.

The calculation was facilitated by using the following series representation for $K_{\alpha}(\tau)[8]:$

$$
\begin{aligned}
K_{\alpha}(\tau)= & 1+\frac{1+4 \alpha^{2}}{4(1 !)^{2}}\left(\frac{1-\tau}{2}\right) \\
& +\frac{\left(1+4 \alpha^{2}\right)\left(3^{2}+4 \alpha^{2}\right)}{4^{2}(2 !)^{2}}\left(\frac{1-\tau}{2}\right)^{2} \\
& +\frac{\left(1+4 \alpha^{2}\right)\left(3^{2}+4 \alpha^{2}\right)\left(5^{2}+4 \alpha^{2}\right)}{4^{3}(3 !)^{2}}\left(\frac{1-\tau}{2}\right)^{3}+\cdots
\end{aligned}
$$


for $|\tau-1|<2$, where appropriate recursive relationships allowed rapid calculation of the series terms for $K_{\alpha}(\tau)$ and its derivatives.

In biological cellular flow systems, especially blood, an arbitrary parameter that is used as a reference to changing shapes is the sphericity index (S.I.) [9]. It is defined as

$$
\text { S.I. }=4.84\left(V^{2 / 3} / S\right),
$$

where $V$ is the volume and $S$ is the surface area of the particle respectively. If $b$ is taken as unity in Eq. (6), the volume and surface area of a spindle are found to be

$$
V=2 \pi\left[a^{2}+2 / 3+a\left(a^{2}+1\right) \sin ^{-1}\left(\frac{1}{a^{2}+1}\right)^{1 / 2}\right]
$$

and

$$
S=4 \pi\left[(\varphi a+1)\left(a^{2}+1\right)^{1 / 2}\right]
$$

where $a=\cot \varphi$.

These values were used in Eq. (17) to calculate the sphericity index as a function of the changing shape of the spindle.

In Table I, the drag coefficient and sphericity index are listed for several different spindles.

A recent paper by Gluckman, Weinbaum and Pfeffer [10] also presents a solution to the problem of axisymmetric slow viscous flow past a convex body of revolution. Although these authors' presentation is thorough and interesting, the unique use of peripolar or bipolar coordinates as suggested by Pell and Payne [13] seems to encompass all of the above authors' bodies as well as several not included in their publication

\section{TABLE I}

Drag coefficients and sphericity indices for spindles

\begin{tabular}{rccc}
\hline \multicolumn{5}{c}{ drag coefficient } & & \\
& \multicolumn{1}{c}{ S. I. } & $D / \mathrm{S} . \mathrm{I}$. \\
\hline 90 & 18.85 & 1.000 & 18.85 \\
95 & 17.86 & 0.999 & 17.88 \\
100 & 16.95 & 0.997 & 17.00 \\
105 & 16.13 & 0.992 & 16.26 \\
110 & 15.38 & 0.985 & 15.61 \\
115 & 14.71 & 0.975 & 15.09 \\
120 & 14.12 & 0.962 & 14.68 \\
125 & 13.59 & 0.947 & 14.35 \\
130 & 13.13 & 0.928 & 14.15 \\
135 & 12.72 & 0.905 & 14.06 \\
140 & 12.37 & 0.879 & 14.07 \\
145 & 12.07 & 0.848 & 14.23 \\
150 & 11.81 & 0.811 & 14.56 \\
155 & 11.60 & 0.768 & 15.10 \\
160 & 11.43 & 0.716 & 15.96 \\
165 & 11.30 & 0.654 & 17.29 \\
170 & 11.20 & 0.572 & 19.58 \\
175 & 11.15 & 0.455 & 24.51 \\
\hline
\end{tabular}


The authors would like to acknowledge the help of Dr. L. E. Payne who introduced us to his work and encouraged us throughout the calculations.

\section{Refarences}

[1] L. E. Payne and W. E. Pell, The Stokes flow problem for a class of axially symmetric bodies, J. Fluid Mech. 7, 529-549 (1960)

[2] L. E. Payne, On axially symmetric flow and the method of generalized electrostatics, Quart. Appl. Math. 10, 197-204 (1952)

[3] W. E. Pell and L. E. Payne, The Stokes flow about a spindle, Quart. Appl. Math. 18, 257-262 (1960)

[4] E. W. Hobson, The theory of spherical and ellipsoidal harmonics, University Press, Cambridge, 1965, p. 451

[5] C. Neumann, Uber die Mehler'schen Kugelfunktionen und deren Anwendung auf electrostatische Probleme, Math. Ann. 18, 195-236 (1881)

[6] M. I. Zhurina and L. N. Karmazina, Tables and formulae for the spherical functions $P_{-1 / 2+i \tau}{ }^{m}(Z)$, Pergamon Press, New York, (1966)

[7] M. I. Zhurina and L. N. Karmazina, Tables of the Legendre functions $P_{-1 / 2+i \tau}(X)$, Part I, Macmillan, New York, 1964

[8] A. Erdelyi, Higher transcendental functions, Vol. 1, McGraw-Hill, New York, 1953, p. 174

[9] P. B. Canham and A. C. Burton, Distribution of size and shape in populations of normal human red cells, Cir. Res. 22, 405-422 (1968)

[10] M. Gluckman, S. Weinbaum and R. Pfeffer, Axisymmetric slow viscous flow past an arbitrary convex body of revolution, J. Fluid. Mech. 55, 677-709 (1972) 